\title{
La valorisation boursière des états financiers des sociétés françaises : pertinence du référentiel IFRS
}

\author{
Denis Cormier \\ CIFO \\ ESG UQAM \\ Samira Demaria \\ Université de Nice-Sophia Antipolis - GREDEG \\ Pascale Lapointe-Antunes \\ Brock University
}

Cette recherche a été réalisée grâce au soutien financier du fonds pour l'éducation et la saine gouvernance de l'Autorité des marchés financiers ( «' 'autorité »), le Conseil de recherche en sciences humaines du Canada (CRSH), PriceWaterhouseCoopers et KPMG. Les informations, renseignements, opinions et avis exprimés au présent article n'engagent que la responsabilité des auteurs. Le contenu de cet article ne reflète pas nécessairement l'opinion de l'Autorité, du $\mathrm{CRSH}$, de PriceWaterhouseCoopers et de KPMG et les erreurs éventuelles relèvent de la responsabilité des auteurs. 


\title{
La valorisation boursière des états financiers des sociétés françaises : \\ pertinence du référentiel IFRS
}

\section{Résumé :}

Cette étude s'intéresse à la valorisation boursière des états financiers des firmes françaises en vertu du référentiel comptable français et après l'adoption des IFRS. Les résultats sont les suivants : Premièrement, il semble que selon le référentiel français, les comptes du bilan ont un pouvoir explicatif de la valeur boursière bien supérieur aux résultats comptables. Deuxièmement, les accruals anormaux ne semblent être valorisés que selon les IFRS, qu'il s'agisse de l'impact sur la valeur boursière ou sur les rendements boursiers. Enfin, les résultats selon IFRS ont un pouvoir d'anticipation de la performance plus élevé que sous le plan comptable général puisque les rendements boursiers reflètent la performance comptable bien avant l'année de la publication du résultat comptable.

Mots clés : gestion des résultats, IFRS, valorisation boursière.

\section{Stock Market Valuation of Financial Statements under IFRS versus Local GAAP:}

\author{
Evidence from France
}

\begin{abstract}
:
This paper investigates the value relevance of financial statements of a sample of French firms comparing pre and post IFRS adoption. First, results show that the balance sheet accounts are more valued than earnings under French GAAP. Second, abnormal accruals appear to be valued only under IFRS, either for stock prices or stock returns. Finally, earnings under IFRS are more predictable by stock market under IFRS than under French GAAP.
\end{abstract}

Key words: Earnings management, IFRS, value relevance. 


\section{Introduction}

L'Union Européenne (UE) a imposé la conversion des états financiers des groupes européens depuis 2005, afin de doter les marchés d'informations comptables et financières de hautes qualités, transparentes et comparables. Depuis le $1^{\mathrm{er}}$ janvier 2005 toutes les firmes européennes appliquent les normes comptables internationales, les International Financial Reporting Standard (IFRS), pour la constitution de leurs comptes consolidés en lieu et place des normes locales. Parmi les pays concernés, la France a été l'un des plus réticents à ce changement (Ernst \& Young, 2006). En effet, la tradition comptable fiscaliste et juridique, de l'information issue du Plan Comptable Général (PCG) tranche avec les normes IFRS financières à connotation économique et destinées prioritairement aux investisseurs. Disle et Noël (2007) mettent en évidence les profondes divergences conceptuelles entre les normes IFRS et le PCG. De même Ding et al. (2007) ont montré que la France est l'un des pays européens dont les normes locales différent le plus des normes IFRS. Dès lors, l'application des IFRS en France aurait dû constituer un changement profond de la nature de l'information.

Les groupes français appliquent les normes IFRS depuis l'exercice 2005, il est donc à présent temps de vérifier si l'objectif initial a été atteint. En effet, les comptes sont- ils plus pertinents selon les IFRS que selon les normes issues du PCG ? Le marché valorise-t-il plus les données issues des nouveaux états financiers en IFRS ? Les comptes en IFRS reflètent-ils mieux la réalité économique ? Ont-ils une valeur prédictive ? Nous souhaitons répondre à ces différentes interrogations afin d'être en mesure de vérifier les effets attendus des normes IFRS. 
Plus précisément, cet article vise à répondre aux deux questions de recherche suivantes : (1) Le passage aux normes IFRS a-t-il amélioré la valorisation des bilans et résultats des groupes français? (2) Les comptes en IFRS ont-ils une valeur prédictive supérieure aux comptes au format du PCG?

La recherche s'appuie sur une période de douze exercices (1997 à 2008). Le choix de la durée d'étude relève de plusieurs intérêts : d'une part l'analyse de douze exercices nous permet de tenir compte des évolutions normatives (passage du $\mathrm{PCG}^{1}$ aux IFRS) et d'avoir un recul sur chacune des deux réglementations (huit ans sous le PCG, et quatre ans sous les IFRS). D'autre part, cela nous permet de limiter les biais liés à la période de changement, en effet avant le changement de normes ou juste après, le marché peut réagir de manière exagérée.

L'étude porte sur les firmes françaises appartenant à l'indice SBF 250. En retenant les firmes non financières du SBF 250, nous étudions un panel d'entreprises bien plus large que seulement les très grands groupes du CAC 40. Le SBF 250 reflète, selon nous, toute la diversité de la mise en application des normes IFRS. C'est véritablement sur ce type d'échantillon que nous serons le plus à même de tirer les conclusions de l'application des normes IFRS.

\footnotetext{
${ }^{1}$ Le changement de PCG en 1999 n'introduit pas de différences significatives dans les éléments comptables étudiés. C'est la raison pour laquelle nous ne différencions pas les normes du PCG avant et après 1999.
} 
Il ressort des résultats les constats suivants : (1) les immobilisations corporelles sont valorisées dans la même proportion selon le PCG et IFRS, les immobilisations incorporelles montrent un multiple inférieur selon IFRS, ce qui est peut-être dû au fait que les soldes sont supérieurs. Par ailleurs, tel qu'anticipé, les soldes de passif d'impôts différés et la part des actionnaires sans contrôle semblent davantage valorisés selon IFRS. Cela indiquerait que ces deux éléments reflètent mieux la réalité économique selon IFRS. Selon le PCG, il est plus difficile d'apprécier la juste valeur de ces deux éléments du bilan; (2) les résultats résiduels (ajoutés à l'équation comptable fondamentale) sont uniquement valorisés en IFRS. Il apparaît que selon le PCG, les comptes du bilan ont un pouvoir explicatif de la valeur boursière bien supérieur aux résultats comptables ; (3) les accruals anormaux ne semblent être valorisés que selon les IFRS, qu'il s'agisse de l'impact sur la valeur boursière ou sur les rendements boursiers. Cela pourrait indiquer que la gestion du résultat sous IFRS renseigne davantage les marchés à propos des perspectives de gains futurs de la firme ; et (4) il ressort de nos analyses que les états financiers sous IFRS ont un pouvoir d'anticipation de la performance plus élevé que sous le plan comptable général puisque les rendements boursiers reflètent la performance comptable bien avant l'année de la publication du résultat comptable.

Cette recherche est la première à confirmer la pertinence et les effets, en termes de valorisation et de prédiction, des normes comptables IFRS. Ce travail contribue à la recherche comptable en démontrant, sur une longue période, la qualité supérieure des normes internationales sur les normes locales. Enfin, elle est particulièrement intéressante pour les normalisateurs et les pays qui hésiteraient à basculer vers les normes IFRS. 
L'étude s'articule comme suit. La section 2 présente le cadre théorique. La méthodologie est décrite à la section 3 . Les résultats empiriques font l'objet de la section 4 alors que la section 5 tient lieu de conclusion.

\section{Cadre théorique}

La revue de la littérature s'articule autour des travaux consacrés à la valorisation du bilan et du résultat (2.1), la valorisation des éléments du compte de résultat (2.2) et l'anticipation du résultat comptable par les marchés boursiers (2.3).

\subsection{Valorisation du bilan et du résultat}

L'étude de Lenormand et Touchais (2009) portant sur 160 groupes français du SBF 120 pour l'exercice 2004, montre que le passage des normes du PCG aux IFRS se traduit globalement par une augmentation importante du bénéfice et de la rentabilité financière et, dans une moindre mesure, des capitaux propres alors même que la réalité économique de l'entreprise ne change pas. Cormier et al. (2009) montrent que l'ajustement initial des fonds propres suite à l'adoption des IFRS par les firmes françaises est plus valorisé par les marchés boursiers que les fonds propres initialement établis selon le référentiel comptable français. Il y a donc lieu de s'attendre à ce que les bilans préparés selon le référentiel IFRS aient un contenu informationnel pour les marchés boursiers supérieur au référentiel français. 
Nous concentrons notre analyse sur trois éléments du bilan mise à part les capitaux propres. Il s'agit des immobilisations corporelles, des immobilisations incorporelles et des passifs d'impôts différés. Ces éléments sont susceptibles de présenter des différences notoires entre le Plan comptable général (PCG) et les IFRS. ${ }^{2}$

Immobilisations corporelles. On peut s'attendre à ce que les règles d'amortissement permises selon le PCG axées sur la fiscalité génèrent des charges d'amortissement supérieures à ce qui est permis selon IFRS. Dès lors, nous anticipons un niveau supérieur d'immobilisations corporelles selon IFRS. À titre d'exemple, le groupe Carrefour a changé l'estimation de la durée d'amortissement de ses constructions, la portant de 20 à 40 ans. Cette décision de mieux rapprocher la charge d'amortissement de l'amortissement économique aura pour effet d'augmenter la valeur aux livres des immobilisations. Pour le groupe Renault, l'ajustement de transition aux normes IFRS a fait passer le montant des immobilisations corporelles nettes de 10 595 millions $€$ à 11597 millions $€$. Enfin, l'étude menée par Marchal et al. (2007) sur un échantillon de 597 groupes français, met en évidence une augmentation moyenne de 3\% du montant des immobilisations corporelles. Par ailleurs, l'amortissement sous IFRS est plus susceptible de représenter la dépréciation économique des actifs. Dans un marché efficient, nous devrions ainsi retrouver un multiple de valorisation inférieur sous le référentiel IFRS si le

\footnotetext{
${ }^{2}$ D'autres comptes du bilan sont susceptibles de différer entre le PCG et les IFRS. Mentionnons la dette de retraite et les instruments financiers. Toutefois, dans ces deux cas de figure, l'information pré IFRS est à toutes fins utiles non disponible car aucune norme de comptabilisation précise n'était prévue dans le PCG.
} 
montant des immobilisations corporelles présenté au bilan est supérieur à celui observé sous le PCG.

Immobilisations incorporelles. Bessieux-Ollier et Walliser (2007) comparent le montant et la nature des actifs incorporels selon les normes françaises et IFRS pour les exercices 2003 à 2005. Leur étude qualitative repose sur l'analyse des rapports annuels des groupes du CAC 40. Les auteurs montrent que de manière générale on constate une évolution de la part des incorporels selon les normes IFRS. Le passage aux IFRS introduit une présentation séparée du goodwill et des autres actifs incorporels identifiables. Les groupes français ont aussi profité du changement de normes pour réévaluer le goodwill et pour activer les dépenses de recherche et développement.

Par exemple, le groupe Carrefour a modifié ses pratiques d'amortissement des incorporels au moment du changement de normes. Ainsi, selon le PCG, les fonds de commerce sont amortis sur 20 ans et les écarts d'acquisitions sur maximum sur 40 ans, alors qu'en IFRS, l'amortissement de l'écart d'acquisitions n'est plus permis. Dès lors, mécaniquement le montant des actifs incorporels augmente. Quant au groupe Renault, l'ajustement de transition aux normes IFRS a fait passer le montant des immobilisations incorporelles nettes de 1969 millions $€$ à 2657 millions $€$. Un montant supérieur au bilan est donc anticipé selon IFRS comparativement au PCG. Si tel est le cas, et que les nouvelles règles comptables n'apportent pas d'information nouvelle aux marchés boursiers, le multiple boursier devrait être plus bas dans un marché efficient. 
Actifs financiers. Selon la norme IAS No. 39, les actifs financiers sont mesurés à leur juste valeur sauf : les titres de dettes et créances ayant pris naissance dans l'entreprise et non acquis pour générer un rendement à court terme (not held for trading); les titres que l'entreprise a l'intention de conserver jusqu'à l'échéance ; tout actif financier dont la juste valeur ne peut être mesurée de façon fiable. Les plus (moins) values dues à un changement dans la juste valeur d'un actif ou passif financier acquis dans une optique de générer un rendement à court terme (held for trading) doivent être constatées dans l'exercice courant. Les plus (moins) values sur actifs financiers destinés à être cédés sont comptabilisées dans les capitaux propres jusqu'à la vente ou la constatation d'une perte de valeur. Comme selon le plan comptable général, les actifs financiers sont comptabilisés au coût historique, nos anticipons que la somme des coefficients Actifs financiers et l'interaction Actifs financiers ${ }^{*} I F R S$ aura tendance à se rapprocher de 1, c'est-à-dire de la valeur de marché.

Passifs d'impôts différés. La norme IAS 12 prévoit la comptabilisation d'impôts différés actifs ou passifs lorsque la valeur comptable d'un actif ou passif diffère de sa valeur fiscale. Étant donné que la charge d'amortissement comptable est susceptible d'être inférieure aux amortissements fiscaux, nous pouvons nous attendre à observer un solde de passif d'impôts différés supérieur selon IFRS que selon le PCG. Par exemple, pour le groupe Carrefour, le changement d'estimation de la durée d'amortissement de ses constructions de 20 à 40 ans a fait passer le solde de passif d'impôts différés de 353 millions $€$ à 397 millions $€$. De plus, il est permis de penser que le solde d'impôts différés sous IFRS représente mieux la réalité économique, i.e. les impôts qui deviendront éventuellement exigibles, car il peut s'avérer 
difficile pour les investisseurs d'estimer l'ampleur des impôts différés non comptabilisés. Dans un marché efficient, les investisseurs devraient tenir compte du fait que sous le PCG, le solde de passif d'impôts différés est sous-évalué par rapport au référentiel IFRS. Dès lors, nous anticipons ainsi un multiple de valorisation inférieur sous IFRS comparativement au PCG.

Résultat. Dans le contexte français, l'étude de Janin (2002), portant sur la période de 1993 à 1998, montre que le résultat net a un contenu informationnel pour expliquer la valeur boursière des firmes françaises. Toutefois, dans une étude comparative France, États-Unis, Suisse, l'étude de Cormier et al. (2001) montre que le contenu informationnel du résultat comptable pour expliquer les rendements boursiers est nettement inférieur pour les firmes françaises que leurs homologues américaines $\left(\mathrm{R}^{2}\right.$ de $3,3 \%$ pour la France, $4,3 \%$ pour la Suisse et $28,2 \%$ pour les États-Unis). Les résultats de l'étude de Cormier et al. (2001) sont quasi-identiques lorsque qu'ils utilisent le résultat résiduel (résultat net - coût du capital) comme indicateur de performance. Dans la présente étude, nous nous intéressons à la question de savoir si le résultat résiduel est pertinent dans le contexte français lorsqu'on tient compte du contenu informationnel du bilan.

\subsection{Valorisation des éléments du compte de résultat}

Quelques études se sont intéressées à la première application des normes comptables internationales. Principalement, c'est la compréhension des déterminants des choix qui ont fait l'objet des études (Cazavan-Jeny et Jeanjean, 2007 ; Cormier et al., 2009). Ces choix sont essentiellement tributaires du niveau d'endettement, de l'impact sur le rendement de l'actif, de la 
taille de la firme, de la cotation à une bourse étrangère, de la présence de blocs de contrôle et de stocks options et du secteur d'activité.

Les IFRS confèrent aux dirigeants d'entreprises une grande marge de manœuvre dans l'élaboration des états financiers. En effet, l'orientation vers la juste valeur repose sur des estimations et prévisions qui émanent de la direction. L'orientation vers la juste valeur dans la mesure des différents comptes des états financiers aura également des conséquences pour les analystes financiers et les investisseurs. Dans ce contexte, nous pouvons nous attendre à observer un niveau d'accruals anormaux supérieur selon le référentiel IFRS. L'étude de Kaserer et Klingler (2008) arrive à ce constat. L'approche de juste valeur préconisée dans les référentiels IFRS et US-GAAP laisse plus de marge de manœuvre aux directions d'entreprises pour gérer le résultat comptable. À cet effet, Christensen et al. (2009) montrent que l'adoption du référentiel IFRS n'est pas un gage de qualité des résultats publiés, surtout quant la firme n'a pas d'intérêt à adopter ce référentiel.

Par ailleurs, selon l'étude de Pincus et al. (2007), l'anomalie des accruals (« accrual anomaly ») serait moins importante dans les pays de droit codifié tel que la France comparativement aux pays de droit coutumier. Si tel est le cas, nous pourrions nous attendre à ce que les accruals anormaux soient davantage valorisés par les marchés boursiers selon IFRS puisque ce référentiel s'apparente dans une large mesure à la comptabilité anglo-saxonne et est très peu axé sur des principes de droit fiscal. 
Landsman et al. (2009) examinent le contenu informationnel pour les marchés boursiers à l'annonce des résultats comptables suite à l'adoption des IFRS. L'échantillon porte sur 16 pays ayant basculé aux IFRS comparés à 11 pays ayant conservé leur référentiel comptable local. Les résultats montrent que le contenu informationnel tel que mesuré par la volatilité anormale des titres à l'annonce des résultats comptables augmente dans les pays étant passés aux IFRS comparativement aux autres pays. Cela se confirme davantage pour les pays scandinaves, l'Allemagne et la France, les pays de droit codifié versus les pays de droit commun. Par ailleurs, Daske et al. (2008), à partir d'une étude comprenant 26 pays ayant adopté IFRS, montrent que la liquidité des titres telle qu'exprimée par l'écart offre-demande s'est améliorée lors de l'adoption. Ce résultat s'observe davantage dans les pays où la réglementation financière est élevée. Dans ce contexte, les firmes ont en effet intérêt à pratiquer la transparence financière. Nous n'effectuons donc pas de prédiction quant à la valorisation des accruals anormaux sous IFRS.

\subsection{Anticipation du résultat comptable par les marchés boursiers}

Si les éléments du compte de résultat ont un pouvoir prédictif, ils seront anticipés par les marchés boursiers bien avant la publication du rapport annuel. Nous postulons que les états financiers sous IFRS ont un pouvoir d'anticipation plus élevé que sous le PCG. Contrairement au PCG, le cadre conceptuel de l'IASB met l'accent sur la pertinence de l'information financière en tant qu'outil de prévision des flux de trésorerie futurs d'une entreprise. Cette orientation vers le futur, le recours à des hypothèses et à des modèles de plus en plus sophistiqués, l'adoption de normes basées sur la juste valeur et des exigences de divulgation accrues découlent directement de la philosophie sous-jacente au cadre conceptuel du référentiel IASB. Cette orientation vers la 
pertinence boursière vise à assurer que les états financiers reflètent la performance et la situation financière des entreprises. Si tel est le cas, les rendements boursiers devraient refléter la performance comptable sous IFRS bien avant l'année de la publication du résultat comptable.

\section{Méthodologie}

Dans cette section, nous détaillerons d'abord l'échantillon (3.1) puis nous aborderons la présentation des modèles. Nous proposons trois modèles : le premier de valorisation du bilan et du résultat (3.2), le deuxième de valorisation des éléments du compte de résultat (3.3) et le troisième d'anticipation du résultat comptable par les marchés financiers (3.3).

\section{1 Échantillon}

L'échantillon porte sur les 217 firmes non financières de l'indice boursier français SBF 250 pour les années 1997 à 2008 pour neuf secteurs d'activité. Les données proviennent de la base de données Compustat. Un échantillon de 217 firmes sur une période de 12 ans représente 2604 observations. Comme plusieurs firmes ont été introduites en bourse après 1997, la cote boursière n'est pas disponible pour 433 observations, ce qui nous amène à 2126 observations. À cause de diverses données manquantes, nous obtenons un échantillon de 1956 observations pour le modèle 1 (bilan et résultat résiduel). Pour le modèle 3, le calcul des rendements boursiers nous fait perdre 214 observations additionnelles, ce qui laisse un échantillon de 1742 observations. Pour le modèle 2, en tenant compte du fait que le calcul des accruals se fonde sur le flux de 
trésorerie de l'année précédente, nous perdons une année d'observations. Nous obtenons un échantillon de 1426 observations.

\subsection{Modèle 1 : Valorisation du bilan et du résultat}

L'équation comptable fondamentale sert de base à plusieurs modèles de valorisation boursière (Fetltham et Ohlson, 1995 ; Amir et Lev, 1996 ; Collins et al., 1999). À l'équation comptable, on ajoute généralement un facteur de croissance. À cet effet, nous calculerons un résultat résiduel et anticipons une relation positive avec la cote boursière. Le résultat résiduel est calculé en appliquant un taux fixe de $8 \%$ aux capitaux propres de toutes les firmes de l'échantillon. Cette approche a été utilisée dans des études antérieures (e.g. Bernard, 1995 ; Ball et al., 2000).

Notre premier modèle empirique se présente comme suit :

(1) Valeur boursière =

$\beta_{0}+\beta_{1}$ (Capitaux propres - Immobilisations corporelles - Immobilisations incorporelles

+ Passif d'impôts différés) ${ }_{\text {it }}+\beta_{2}$ Immobilisations corporelles it $+\beta_{3}$ Immobilisations incorporelles $_{\text {it }}+\beta_{4}$ Actifs financiers ${ }_{\text {it }}+\beta_{5}$ Passif d'impôts différés ${ }_{i t}+\beta_{6}$ Résultat résiduel it $+\beta_{7}$ Immobilisations corporelles*IFRS $_{\text {it }}+\beta_{8}$ Immobilisations incorporelles* $^{*}$ IFRS $_{\text {it }}+$ $\beta_{9}$ Actifs financiers* $*$ IFRS $_{\text {it }}+\beta_{10}$ Passif d'impôts différés*IFRS ${ }_{\text {it }}+\beta_{11}$ Résultat résiduel*IFRS ${ }_{\text {it }}+\beta_{12}$ IFRS $+\varepsilon$ 
Toutes les variables sont normalisées par le nombre d'actions en circulation en fin de période. Les termes d'interaction servent à évaluer dans quelle mesure le référentiel IFRS affecte la valorisation boursière des éléments du bilan et le facteur de croissance exprimé par le résultat résiduel.

Comme nous utilisons des données de panels, nous estimons les régressions par la méthode des moindres carrés généralisés. Le test de Breusch-Pagan / Cook-Weisberg montre la présence d'hétéroscédasticité. Ainsi, la structure des erreurs parmi les panels sera présumée hétéroscédastique.

\subsection{Modèle 2 : Valorisation des éléments du compte de résultat}

Pour analyser les différences de valorisation boursière des éléments du compte de résultat, nous allons d'abord subdiviser le résultat en trois composantes : flux de trésorerie d'exploitation ; accruals normaux ; et accruals anormaux. Nous calculons les accruals normaux par secteur d'activité et pour les 12 années d'observations combinées.

Nous mesurons la gestion des résultats à partir des accruals expliquant la différence entre le résultat net et le flux de trésorerie d'exploitation. Selon Hribar et Collins (2002), cette façon de mesurer les accruals est préférable à l'approche fondée sur le bilan, laquelle peut créer un biais systématique dans l'estimation de la portion anormale des accruals. Les auteurs montrent que l'estimation des accruals est systématiquement biaisée en présence d'opérations de fusions et 
acquisitions pour l'échantillon d'estimation. Pour une firme i et une période t donnée, les accruals normaux sont calculés de la manière suivante :

$$
\text { Accruals }_{\text {it }}=\alpha_{1} \Delta \mathrm{CA}_{\text {it }}+\alpha_{2} \mathrm{FTE}_{\text {it- } 1}+\alpha_{3} \mathrm{IMMO}_{\text {it }}+\varepsilon_{\text {it }}
$$

De la littérature antérieure trois variables clés ressortent comme déterminants des acrruals normaux : (1) la performance captée par le chiffre d'affaires, (2) les immobilisations, (3) le flux de trésorerie d'exploitation antérieur (e.g. Dechow et al. 1994; Erickson et Wang, 1999) ou les accruals antérieurs (Beneish, 1997; Defond et Park, 1997, 2001). L’équation précédente indique que le montant des accruals est fonction de la variation du chiffre d'affaires $(\Delta \mathrm{CA})$, du flux de trésorerie généré par l'exploitation en t-1 $\left(\mathrm{FTE}_{\mathrm{it}-1}\right)$ et des immobilisations en t $\left(\mathrm{IMMO}_{\mathrm{it}}\right)$. En continuité avec l'équation comptable fondamentale, Dechow (1994) propose de considérer le flux de trésorerie d'exploitation comme un facteur déterminant le niveau de variables comptables de régularisation non discrétionnaires. La pertinence de cette variable se justifie par la corrélation négative très forte observée entre le flux de trésorerie d'exploitation d'une période et le niveau d'accruals de la période suivante. Dans la mesure où une portion importante du niveau des accruals semble prédéterminée par le flux de trésorerie d'exploitation de l'exercice précédent, il est logique de penser qu'il s'agit d'une portion non discrétionnaire.

Le modèle d'accruals anormaux est estimé par une régression temporelle de coupe instantanée au sens des moindres carrés sur une période de 12 ans pour chacun des 9 secteurs d'activité (1997- 
2008). Comme le modèle d'estimation utilise le flux de trésorerie de l'année antérieure, cela nous laisse 11 années d'observations. Le nombre d'observations par secteur d'activité varie de 41 pour le secteur du service public (utilities) à 639 pour le secteur industriel. Le total d'observations années pour les accruals normaux et de 1936.

Notre modèle 2 se présente comme suit :

(2) Valeur boursière =

$$
\begin{aligned}
& \beta_{0}+\beta_{1} \text { Flux de trésorerie d'exploitation }_{\text {it }}+\beta_{2} \text { Accruals normaux }_{\text {it }}+\beta_{3} \text { Accruals } \\
& \text { anormaux }_{\text {it }}+\beta_{4} \text { Accruals normaux }^{*} \text { IFRS }_{\text {it }}+\beta_{5} \text { Accruals anormaux*IFRS }_{\text {it }}+\beta_{5} \text { IFRS }_{\text {it }} \\
& +\varepsilon
\end{aligned}
$$

Toutes les variables sont normalisées par le nombre d'actions en circulation en fin de période. Nous estimons les régressions par la méthode des moindres carrés généralisés. Le test de Breusch-Pagan / Cook-Weisberg montre la présence d'hétéroscédasticité. Ainsi, la structure des erreurs parmi les panels sera présumée hétéroscédastique.

Les termes d'interaction servent à évaluer dans quelle mesure le référentiel IFRS affecte la valorisation boursière des éléments du compte de résultat. Les études antérieures montrent généralement une augmentation du contenu informationnel des états financiers selon IFRS, surtout pour les pays à forte réglementation financière. 


\subsection{Modèle 3 : Anticipation du résultat comptable par les marchés boursiers}

En accord avec l'étude de Warfield et Wild (1992), le modèle de régression suivant est estimé :

(3) Rendement des fonds propres $=$

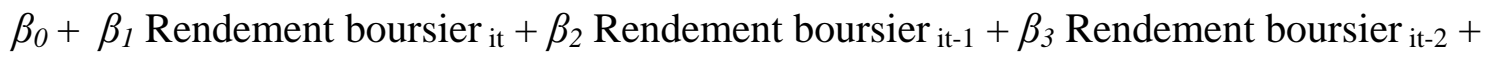
$\beta_{4}$ Rendement boursier*IFRS $_{\text {it }}+\beta_{5}$ Rendement boursier*IFRS ${ }_{\mathrm{it}-1}+\beta_{6}$ Rendement boursier*IFRS $_{\mathrm{it}-2}+\beta_{7}$ IFRS $+\varepsilon$

Nous estimons les régressions par la méthode des moindres carrés généralisés. Le test de Breusch-Pagan / Cook-Weisberg montre la présence d'hétéroscédasticité. Dès lors, la structure des erreurs parmi les panels sera présumée hétéroscédastique.

\section{Résultats}

Les résultats seront présentés de la façon suivante: d'abord les résultats du modèle de valorisation du bilan (4.1), puis ceux du modèle de valorisation du compte de résultat (4.2) et enfin les résultats du modèle d'anticipation du résultat comptable par le marché financier (4.3). 


\subsection{Valorisation du bilan et du résultat}

\subsubsection{Statistiques descriptives}

Les résultats présentés aux tableaux 1 et 2 montrent que les immobilisations corporelles, les immobilisations incorporelles et les impôts différés passifs sont de taille plus élevée selon IFRS et leur corrélation avec la valeur boursière, sauf pour les immobilisations corporelles, est nettement supérieure comparativement au PCG.

\subsubsection{Analyses multivariées}

Le tableau 3 présente les résultats de la régression temporelle de coupe instantanée au sens des moindres carrés généralisés. Il ressort des résultats que les immobilisations corporelles sont valorisées dans la même proportion selon le PCG et IFRS puisque le coefficient pour le terme d'interaction Immobilisations corporelles $*$ IFRS n'est pas significatif $(-0,07 ; \mathrm{p}<0,196)$, les immobilisations incorporelles montrent un multiple inférieur selon IFRS $(-0,21 ; p<0,004)$, ce qui est peut-être dû au fait que les soldes sont supérieurs. Si les soldes se rapprochent davantage de la juste valeur selon IFRS, nous pouvons nous attendre à ce que la somme des coefficients Immobilisations corporelles et Immobilisations corporelles*IFRS s'approche de 1,0, ce qui est le cas. Dans le cas des actifs financiers, étant donné qu'ils sont comptabilisés selon la règle du plus bas du coût d'acquisition ou de la juste valeur selon le référentiel français, nous anticipons un coefficient inférieur à 1,0 pour la variable Actifs financiers et un coefficient positif pour le terme d'interaction Actifs financiers *IFRS. Nos résultats vont dans ce sens (coefficient de la variable Actifs financiers 0,$77 ; \mathrm{p}<0,000$ et Actifs financiers $* I F R S 0,45 ; \mathrm{p}<0,039)$. Par ailleurs, tel 
qu'anticipé, le multiple de valorisation du solde d'impôts différés passif est inférieur selon IFRS en comparaison avec le PCG $(-2,29+1,60=-0,69$ versus $-2,29)$. Il ressort de ce résultat que le marché boursier français sous le PCG appliquait un multiple aux impôts différés de plus de trois fois plus élevé que selon IFRS. Le marché était donc en position de détecter une sous-évaluation en la matière. Par ailleurs, sous le référentiel IFRS, les coefficients des comptes du bilan ont plus tendance à se rapprocher de 1,0 que sous le PCG. Un coefficient de 1,0 signifie que la valeur comptable d'un compte du bilan correspond à sa valeur boursière.

Il ressort également du tableau 3 que dans l'équation comptable fondamentale, les résultats (résultats résiduels) sont davantage valorisés en IFRS $(4,16 ; \mathrm{p}<0,000)$. Il apparaît que selon le PCG, les comptes du bilan ont un pouvoir explicatif de la valeur boursière supérieur aux résultats comptables. Ce constat nous conforte quant à la pertinence de notre deuxième volet de recherche, soit l'étude des différences de valorisation des éléments du compte de résultat.

À titre d'analyse de sensibilité, nous avons réestimé le modèle de régression en enlevant l'année 2008. L'année 2008 a connu de lourdes pertes boursières (médiane de -44\% de rendement), ce qui contraste avec les autres années post IFRS. Les résultats (non présentés) demeurent similaires en excluant l'année 2008 des analyses pour la valorisation des éléments du bilan. En revanche, le résultat résiduel n'est valorisé que selon le référentiel IFRS. Cela nous amène à exclure l'année 2008 des analyses subséquentes. L'année 2008 est une année de forte diminution des résultats (la moyenne des résultats par action a chuté de $14 \%$ par rapport à 2007 passant de $3,19 €$ à $2,81 €$ ). Les accruals normaux, calculés sur une moyenne de 12 années par secteur d'activité, peuvent ne 
pas être représentatifs de l'année 2008. Une partie des accruals classés comme normaux sont peut-être dans les faits anormaux.

\subsection{Valorisation des éléments du compte de résultat}

\subsubsection{Statistiques descriptives}

Il ressort des résultats présentés au tableau 4 que la période suivant l'adoption des IFRS (20052008), est plus prospère sur le plan économique que la période pré IFRS (1997-2004). La médiane des résultats comptables est près du double, passant de $1,11 €$ par action 1,92€ par action. Au tableau 5, nous présentons l'évolution des éléments du résultat comptable par année. Les résultats comptables ont augmenté en moyenne de $25 \%$ post IFRS (2005-2008) alors qu'ils ont été plutôt stables (+ 5\%) pour la période de 1997-2004.

Nous observons un niveau d'accruals anormaux supérieur selon IFRS comparativement au PCG $(0,23 €$ par action versus $0,05 €)$. Le fait que l'amortissement des écarts d'acquisition ne soit pas permis selon IFRS est certes un élément qui réduit le niveau d'accruals normaux et le résultat net. Par contre, d'autres éléments tels que la charge de retraite sont susceptibles d'augmenter le niveau d'accruals normaux et le résultat net selon IFRS. En terme brut, nous observons une augmentation des accruals normaux selon IFRS (médiane de $-1,25 €$ par action versus -1,52€ par action). 


\subsubsection{Analyses multivariées}

Le tableau 6 présente les résultats de la régression de valorisation des éléments du résultat. Selon le référentiel français (PCG), tant les accruals normaux qu'anormaux sont valorisés par les marchés boursiers. Les accruals normaux semblent moins valorisés selon IFRS que PCG alors que nous observons l'inverse pour les accruals anormaux puisque le coefficient pour le terme d'interaction Accruals normaux*IFRS est négatif et significatif $(-0,86 ; \mathrm{p}<0,004)$ alors que le coefficient pour le terme d'interaction Accruals anormaux*IFRS est positif et significatif (1,05; $\mathrm{p}<0,036$ ). Est-ce à dire que la gestion du résultat sous IFRS renseignerait les marchés à propos des perspectives de gains futurs de la firme?

Au tableau 7, nous présentons les résultats fondés sur les rendements boursiers plutôt que sur la cote boursière. Dans ce modèle, nous regroupons les flux de trésorerie d'exploitation et les accruals normaux pour obtenir le résultat dit normal. Les résultats normaux et $(0,47 ; p<0,000)$ anormaux $(0,41 ; p<0,000)$ semblent davantage valorisés selon IFRS. En cohérence avec les résultats fondés sur la cote boursière, les accruals anormaux ne semblent pas reliés aux rendements boursiers que selon IFRS puisque seul le coefficient pour le terme d'interaction Accruals anormaux*IFRS est statistiquement significatif. 


\subsection{Anticipation du résultat comptable par les marchés boursiers}

\subsubsection{Statistiques descriptives}

L'analyse de corrélation présentée au tableau 8 montre qu'autant les résultats nets (rendement des fonds propres) que le résultat normal (accruals normaux) et anormal (accruals anormaux) sont davantage anticipés selon IFRS. Les résultats comptables et leurs composantes semblent être plus prévisibles selon le référentiel IFRS que selon le PCG.

\subsubsection{Analyses multivariées}

Les résultats de la régression présentés au tableau 9 confirment ce constat selon lequel les résultats comptables et leurs composantes semblent être plus prévisibles selon le référentiel IFRS que selon le PCG. En effet, les rendements boursiers antérieurs selon IFRS semblent prédire la performance future de la firme établie selon IFRS dans une plus grande mesure que la performance établie selon le PCG. En revanche, en ce qui concerne le rendement de l'année courante, le test de différence des coefficients n'est pas significatif $(0,01 ; p<0,94)$. Dès lors, sauf pour l'année courante, les rendements boursiers montrent un pouvoir d'anticipation du résultat comptable en IFRS supérieur au résultat comptable en PCG. Ces résultats montrent la valeur prédictive des résultats comptables sous IFRS.

Le modèle des moindres carrés généralisés tient compte de la présence d'hétéroscédasticité et de l'autocorrélation contemporaine. Toutefois, l'autocorrélation temporelle peut toujours constituée 
un problème. À titre d'analyse de sensibilité, nous ajoutons au modèle de régression le rendement des fonds propres de l'année précédente. Les résultats (non présentés) montrent que le coefficient de la variable Rendement des fonds propres t-1 est positif et significatif alors que les autres coefficients de la régression demeurent quasi similaires à ceux présentés au tableau 9. Cette analyse conforte nos résultats.

\section{Conclusion}

Dans cette étude, nous tentons de répondre aux deux questions de recherche suivantes : (1) Le passage aux normes IFRS a-t-il amélioré la valorisation des bilans et résultats des groupes français? (2) Les comptes en IFRS ont-ils une valeur prédictive supérieure aux comptes au format du PCG ?

Les résultats montrent que selon le référentiel français, les comptes du bilan ont un pouvoir explicatif de la valeur boursière bien supérieur aux résultats comptables. Par ailleurs, les accruals anormaux ne semblent être valorisés que selon les IFRS, qu'il s'agisse de l'impact sur la valeur boursière ou sur les rendements boursiers. Enfin, les états financiers préparés selon le référentiel IFRS ont un pouvoir d'anticipation de la performance plus élevé que les états financiers établis selon le plan comptable général puisque les rendements boursiers reflètent la performance comptable bien avant l'année de la publication du résultat comptable. 
Le cadre conceptuel de l'IASB met l'accent sur la pertinence de l'information financière en tant qu'outil de prévision des flux de trésorerie futurs d'une entreprise. Cette orientation vers le futur, le recours à des hypothèses et à des modèles de plus en plus sophistiqués et l'adoption de normes basées sur la juste valeur découlent directement de la philosophie sous-jacente au cadre conceptuel du référentiel IASB. Cette orientation vers la pertinence boursière vise à assurer que les états financiers reflètent la performance et la situation financière des entreprises. Les résultats de la présente étude tendent à démontrer que l'information financière issue des IFRS est plus pertinente pour les marchés boursiers que celle issue du plan comptable général.

Toutefois, comme plusieurs études récentes l'ont montré, le référentiel IFRS confère aux dirigeants d'entreprises une grande marge de manœuvre dans l'élaboration des états financiers (Jeanjean et Stolowy, 2008 ; Christensen et al, 2009). En effet, l'orientation vers la juste valeur préconisée par les IFRS repose sur des estimations et prévisions qui émanent de la direction. Cela entraîne une plus grande volatilité dans les résultats publiés par les entreprises, ce qui n'est pas sans conséquences pour les analystes financiers et les investisseurs. 


\section{Tableau 1}

\section{Statistiques descriptives}

\section{(En $€$ par action)}

\begin{tabular}{lrrrrr}
\hline & Min. & Max. & Moyenne & Médiane & Écart type \\
\hline PCG & & & & & \\
Immobilisations corporelles & 0 & 362,75 & 14,64 & 5,62 & 7555 \\
Immobilisations incorporelles & 0 & 885,23 & 13,95 & 4,22 & 4397 \\
Actifs financiers & 0 & 167,63 & 2,22 & 0,39 & 9,28 \\
Impôts différés passifs & -1 & 319,60 & 1,32 & 0,02 & 587 \\
IFRS & & & & & \\
Immobilisations corporelles & 0 & 199,91 & 16,30 & 6,34 & 9455 \\
Immobilisations incorporelles & 0 & 233,64 & 16,07 & 8,61 & 6211 \\
Actifs financiers & $-0,02$ & 63,98 & 1,42 & 0,24 & 4,72 \\
Impôts différés passifs & 0 & 786,56 & 4,18 & 0,55 & 1115 \\
\hline
\end{tabular}




\section{Tableau 2}

\section{Corrélations}

Cours boursiers

\begin{tabular}{lcc}
\hline & Plan comptable & IFRS** \\
\hline Immobilisations corporelles & $0,45^{*}$ & $0,51^{*}$ \\
Immobilisations incorporelles & $0,40^{*}$ & $0,30^{*}$ \\
Actifs financiers & $0,17^{*}$ & $0,55^{*}$ \\
Passif d'impôts différés & $0,11^{*}$ & $0,44^{*}$ \\
\hline
\end{tabular}


Tableau 3

Régression temporelle de coupe instantanée au sens des moindres carrés généralisés Valorisation du bilan et du résultat

\begin{tabular}{lccc}
\hline Variable expliquée $:$ Cours boursier & $\begin{array}{c}\text { Signe } \\
\text { prévu }\end{array}$ & Coefficient & Valeur p* \\
\hline Variables explicatives ${ }^{* *}:$ & & & \\
\hline Fonds propres & + & 1,02 & 0,000 \\
Immobilisations corporelles & + & 0,93 & 0,000 \\
Immobilisations incorporelles & + & 1,38 & 0,000 \\
Actifs financiers & + & 0,77 & 0,000 \\
Passif d'impôts différés & - & $-2,29$ & 0,000 \\
Immobilisations corporelles*IFRS & $+/-$ & $-0,07$ & 0,196 \\
Immobilisations incorporelles*IFRS & $+/-$ & $-0,21$ & 0,004 \\
Actifs financiers*IFRS & $+/-$ & $-0,45$ & 0,039 \\
Passif d'impôts différés*IFRS & $+/-$ & 1,60 & 0,005 \\
Résultat résiduel & + & 0,65 & 0,001 \\
Résultat résiduel *IFRS & + & 4,16 & 0,000 \\
Années IFRS & $?$ & 1,98 & 0,000 \\
\hline
\end{tabular}

$\mathrm{N}=1956$

Test de Wald $=3405(0,000)$

*Test unidirectionnel s'il y a prédiction de signe, bidirectionnel autrement.

**Normalisées par le nombre d'actions. 


\section{Tableau 4}

Statistiques descriptives

\section{Éléments du compte de résultat}

(En $€$ par action)

\begin{tabular}{lrrcrr}
\hline & Min. & Max. & Moyenne & Médiane & Écart type \\
\hline PCG & & & & & \\
\hline $\begin{array}{l}\text { Flux de trésorerie } \\
\text { d'exploitation }\end{array}$ & -502 & 896 & 5,77 & 2,47 & 46,52 \\
Accruals normaux & -36 & 3,14 & $-1,94$ & $-1,25$ & 2,31 \\
Accruals anormaux & -8 & 3,35 & -0.16 & 0,05 & 1,04 \\
Résultat net & -524 & 110 & 0,68 & 1,11 & 20,92 \\
\hline IFRS & & & & & \\
\hline Flux de trésorerie & -40 & 843 & 8,09 & 3,48 & 46,01 \\
d'exploitation & & & & & \\
Accruals normaux & -630 & 0,98 & $-3,30$ & $-1,52$ & 22,48 \\
Accruals anormaux & -9 & 9 & 0,23 & 0,23 & 1,52 \\
Résultat net & -36 & 67 & 2,98 & 1,92 & 5,93 \\
\hline
\end{tabular}




\section{Tableau 5}

Médiane par année

\begin{tabular}{|c|c|c|c|c|c|}
\hline & $\begin{array}{r}\text { Flux de } \\
\text { trésorerie par } \\
\text { action }\end{array}$ & $\begin{array}{r}\text { Accruals } \\
\text { normaux par } \\
\text { action }\end{array}$ & $\begin{array}{r}\text { Accruals } \\
\text { anormaux par } \\
\text { action }\end{array}$ & $\begin{array}{r}\text { Résultat net } \\
\text { par action } \\
\text { (\% variation) }\end{array}$ & $\begin{array}{r}\text { Rendement } \\
\text { boursier }\end{array}$ \\
\hline \multicolumn{6}{|l|}{ PCG } \\
\hline 1998 & 2,33 & $-1,46$ & 0,05 & $0,99(-0,07)$ & 0,15 \\
\hline 1999 & 2,54 & $-1,34$ & 0,08 & $1,15(0,16)$ & 0,24 \\
\hline 2000 & 2,60 & $-1,29$ & 0,08 & $1,26(0,09)$ & 0,00 \\
\hline 2001 & 2,57 & $-1,17$ & 0,09 & $1,02(-0,23)$ & $-0,09$ \\
\hline 2002 & 2,32 & $-1,25$ & 0,02 & $1,17(0,15)$ & $-0,15$ \\
\hline 2003 & 2,22 & $-1,18$ & $-0,03$ & $1,14(0,03)$ & 0,27 \\
\hline 2004 & 2,47 & $-1,20$ & 0,03 & $0,96(0,18)$ & 0,24 \\
\hline 1998-2004 & 2,47 & $-1,25$ & 0,05 & $1,11(0,04)$ & 0,09 \\
\hline \multicolumn{6}{|l|}{ IFRS } \\
\hline 2005 & 3,03 & $-1,32$ & 0,13 & $1,91 \quad(0,90)$ & 0,30 \\
\hline 2006 & 3,60 & $-1,47$ & 0,27 & $1,87(-0,02)$ & 0,21 \\
\hline 2007 & 3,50 & $-1,56$ & 0,29 & $1,92(0,03)$ & 0,07 \\
\hline 2008 & 3,55 & $-1,64$ & 0,29 & $2,09(0,09)$ & $-0,44$ \\
\hline $2005-2008$ & 3,48 & $-1,52$ & 0,23 & $1,92(0,25)$ & 0,06 \\
\hline
\end{tabular}




\section{Tableau 6}

Régression temporelle de coupe instantanée au sens des moindres carrés généralisés

\section{Valorisation des éléments du résultat}

(Cours boursiers)

(Sans l’année 2008)

\begin{tabular}{lccc}
\hline Variable expliquée : Cours boursier & $\begin{array}{c}\text { Signe } \\
\text { prévu }\end{array}$ & Coefficient & Valeur p $^{*}$ \\
\hline Variables explicatives** : & & & \\
\hline Fonds propres & + & 0,78 & 0,000 \\
Flux de trésorerie d'exploitation & + & 3.60 & 0,000 \\
Accruals normaux & + & 3.88 & 0,000 \\
Accruals anormaux & + & 3.32 & 0,000 \\
Accruals normaux*IFRS & $+/-$ & $-0,86$ & 0,004 \\
Accruals anormaux*IFRS & $+/-$ & 1,05 & 0,036 \\
Année IFRS & $+/-$ & 1,25 & 0,393 \\
\hline
\end{tabular}

$\mathrm{N}=1678$

Test de Wald $=4060(0,000)$

* Test unidirectionnel s'il y a prédiction de signe, bidirectionnel autrement.

** Normalisées par le nombre d'actions 


\section{Tableau 7}

Régression temporelle de coupe instantanée au sens des moindres carrés généralisés

\section{Valorisation des résultats normaux et anormaux}

(Rendements boursiers)

(Sans l’année 2008)

\begin{tabular}{lccc}
\hline Variable expliquée : Rendement boursier & $\begin{array}{c}\text { Signe } \\
\text { prévu }\end{array}$ & $\begin{array}{r}\text { Coefficient } \\
\text { Variables explicatives** }:\end{array}$ & $\begin{array}{r}\text { Valeur } \\
\mathrm{p}^{*}\end{array}$ \\
\hline Résultat avant accruals anormaux & + & 0,02 & 0,141 \\
Accruals anormaux & $+/-$ & $-0,03$ & 0,230 \\
Résultat avant accruals anormaux*IFRS & $+/-$ & 0,47 & 0,000 \\
Accruals anormaux*IFRS & $+/-$ & 0,41 & 0,000 \\
Année IFRS & $+/-$ & 0,03 & 0,009 \\
\hline
\end{tabular}

$\mathrm{N}=1595$

Test de Wald $=264.2(0,000)$

* test unidirectionnel s'il y a prédiction de signe, bidirectionnel autrement.

**Normalisées par les fonds propres 


\section{Tableau 8}

\section{Corrélations}

\section{Anticipation des résultats par les marchés boursiers}

(Normalisés par les fonds propres)

\begin{tabular}{|c|c|c|c|}
\hline & Résultat & $\begin{array}{l}\text { Résultat avant } \\
\text { accruals anormaux }\end{array}$ & Accruals anormaux \\
\hline \multicolumn{4}{|l|}{ PCG } \\
\hline $\begin{array}{l}\text { Rendement } \\
\text { boursier } t\end{array}$ & $0,173 *$ & $0,147^{*}$ & 0,019 \\
\hline $\begin{array}{l}\text { Rendement } \\
\text { boursier } t-1\end{array}$ & $0,052 *$ & 0,051 & $0,068 *$ \\
\hline $\begin{array}{l}\text { Rendement } \\
\text { boursier } \mathrm{t}-2\end{array}$ & 0,027 & 0,014 & 0,038 \\
\hline \multicolumn{4}{|l|}{ IFRS } \\
\hline $\begin{array}{l}\text { Rendement } \\
\text { boursier } t\end{array}$ & $0,262 *$ & $0,277 *$ & $-0,022$ \\
\hline $\begin{array}{l}\text { Rendement } \\
\text { boursier t-1 }\end{array}$ & $0,239 *$ & $0,174^{*}$ & $0,058 *$ \\
\hline $\begin{array}{l}\text { Rendement } \\
\text { boursier t-2 }\end{array}$ & $0,110^{*}$ & 0,038 & $0,089 *$ \\
\hline
\end{tabular}




\section{Tableau 9}

Régression temporelle de coupe instantanée au sens des moindres carrés généralisés

\section{Anticipation des résultats par les marchés boursiers}

(Rendement des fonds propres pour l'année t)

\begin{tabular}{|c|c|c|c|c|c|}
\hline $\begin{array}{l}\text { Variable expliquée : Rendement des } \\
\text { fonds propres pour l'année } t\end{array}$ & & $\begin{array}{l}\text { Ensemble de } \\
\text { l'échantillon }\end{array}$ & Année $\mathrm{t}$ & Année t-1 & Année t-2 \\
\hline Variables explicatives: & $\begin{array}{l}\text { Signe } \\
\text { prévu }\end{array}$ & & & & \\
\hline Rendement boursier $\mathrm{t}$ & + & $* * 0,022$ & $* * * 0,016$ & & \\
\hline Rendement boursier t-1 & + & $* * 0,053$ & & $* * * 0,003$ & \\
\hline Rendement boursier $\mathrm{t}-2$ & + & $* 0,002$ & & & $* * 0,003$ \\
\hline Rendement boursier $t^{*}$ IFRS & $+/-$ & $* 0,026$ & $* * 0,017$ & & \\
\hline Rendement boursier t- $1 *$ IFRS & $+/-$ & $-0,020$ & & $* * * 0,033$ & \\
\hline Rendement boursier $\mathrm{t}-2$ *IFRS & $+/-$ & $* * * 0,024$ & & & $* * * 0,019$ \\
\hline Année IFRS & $+/-$ & $* 0,012$ & $* * * 0,026$ & $* * * 0,024$ & $* * * 0,031$ \\
\hline \multicolumn{6}{|l|}{ Test de différence de coefficient } \\
\hline $\begin{array}{l}\text { Rendement boursier et Rendement } \\
\text { boursier *IFRS }\end{array}$ & & & $0,01(0,94)$ & $23,1(0,00)$ & $7,93(0,00)$ \\
\hline Ri versus Ri*IFRS & & $0,10(0,75)$ & & & \\
\hline Ri-1 versus Ri-1*IFRS & & $5,33(0,00)$ & & & \\
\hline $\mathrm{Ri}-2$ versus $\mathrm{Ri}-2 * \mathrm{IFRS}$ & & $8,27(0,00)$ & & & \\
\hline $\mathrm{N}$ & & 1413 & 1713 & 1622 & 1426 \\
\hline Test de Wald & & $101,2(0,00)$ & $108,3(0.00)$ & $195,3(0,00)$ & $106,5(0,00)$ \\
\hline
\end{tabular}

$*: \mathrm{p}<0.10 ; *^{* *}: \mathrm{p}<0.05 ; * * * \mathrm{p}<0.01$. Unidirectionnel s'il y a prédiction de signe, bidirectionnel autrement. 


\section{Bibliographie}

Amir, E. et Lev, M. (1996), Value-Relevance of Non Financial Information: The Wireless Communication Industry, Journal of Accounting and Economics, 22(1-3), 3-30.

Ball, R., Kothari, S.P. et Robin, A. (2000), The Effect of International Institutional Factors on Properties of Accounting Earnings, Journal of Accounting and Economics, 29(1), 1-52.

Bernard, V. (1995), The Feltham-Ohlson Framework: Implications for Empiricists.

Contemporary Accounting Research, 10(2), 733-747.

Beneish, M.D. (1997), Detecting GAAP Violation: Implications for Assessing Earnings Management among Firms with Extreme Financial Performance, Journal of Accounting and Public Policy, 16(3), 271-309

Bessieux-Ollier, C et Walliser, E. (2007), La transition et le bilan de la première application en France des normes IFRS L Le cas des incorporels, Comptabilité-Contrôle-Audit, supplément, 219-246.

Cazavan-Jeny, A. et Jeanjean, T. (2007), Accounting Choices under IFRS 1: Analysis and

Determinants, Accounting in Europe $2^{\text {rd }}$ annual Workshop, septembre.

Cazavan-Jeny, A. et Jeanjean, T (2009). Il faut tout changer pour que rien ne change, Comptabilité contrôle audit, 15(1), 105-131

Christensen, H.B., Lee, E. et Walker, M. (2009), Do IFRS Reconciliations Convey Information? The Effect of Debt Contracting, Journal of Accounting Research, 47(5), 1167-1199.

Collins, D., Pincus, M. et Xie, H. (1999) Equity Valuation and Negative Earnings: The Role of Book Value of Equity. The Accounting Review, 74(1), 29-61.

Cormier, D., Demaria, S., Lapointe et Teller, R (2009), First-Time Adoption of IFRS, Managerial Incentives and Value-Relevance: Some French Evidence, Journal of International Accounting Research, 8(2), 1-22. 
Cormier, D., Magnan, M. et Zéghal, D. (2001), La pertinence et l'utilité prédictive des mesures de performance financière : une comparaison France, États-Unis et Suisse», ComptabilitéContrôle-Audit, 6(1), 77-105.

Daske, H., Hail, L., Leuz, C. et Verdi, R. (2008), Mandatory IFRS Reporting around the World: Early Evidence on the Economic Consequences, Journal of Accounting Research, à paraître.

Dechow, P. (1994), Accounting Earnings and Cash Flows as Measures of Firm Performance: The Role of Accounting Accruals, Journal of Accounting and Economics, 18(1), 3-42.

Defond, M.L. et Park, C.W. (1997), Smoothing Income in Anticipation of Future Earning, Journal of Accounting and Economics, 23, 115-139.

DeFond, M.L. et Park, C.W. (2001), The Reversal of Abnormal Accruals and the Market Valuation of Earnings Surprises, The Accounting Review, 76(3), 374-404.

Ding, Y., Hope, O-K., Jeanjean, T. et Stolowy, H. (2007), Differences between Domestic Accounting Standards and IAS: Measurement, Determinants, and Implications. Journal of Accounting and Public Policy, 26(1): 1-38.

Disle, C et Noël C. (2007), La révolution des normes IFRS Une convergence de la comptabilité vers la finance ?, La Revue des Sciences de Gestion, Direction et Gestion n²24-225, 17-27

Erickson, M. et Wang, S. (1999), Earnings Management by Acquiring Firms in Stock for Stock Mergers, Journal of Accounting and Economics, 27, 149-176.

Ernst \& Young (2006), Première application des IFRS, CPC éditions, Paris.

Feltham, G. et Ohlson, J. (1995), Valuation and Clean Surplus Accounting for Operating and Financial Activities, Contemporary Accounting Research, 11(2), 689-731.

Hribar, P. et Collins, D.W. (2002), Errors in Estimating Accruals: Implications for Empirical Research, Journal of Accounting Research, 40, 105-134.

Janin, R. (2002), Les contenus informationnels respectifs du résultat net et des indicateurs de flux de trésorerie dans le contexte français, Comptabilité-Contrôle-Audit, 2(8), 69-85. 
Jeanjean, T et Stolowy, H. (2008), Do Accounting Standards Matter? An Exploratory Analysis of Earnings Management before and after IFRS Adoption, Journal of accounting and public policy, 27(6): 480-494

Kaserer, C. et Klingler, C. (2008), The Accrual Anomaly under Different Accounting Standards - Lessons Learned from the German Experiment, Journal of Business Finance \& Aaccounting, 35 (7/8), 837-859.

Landsman, W.R., Maydew, E.L. et Thornock, J.R. (2009), The Information Content of Annual Earnings Announcements and Mandatory Adoption of IFRS. SSRN: http://ssrn.com/abstract=1337567

Lenormand, G et Touchais, L (2009), Les IFRS améliorent-elles la qualité de l'information financière ? Approche par la value relevance, Comptabilité Contrôle Audit, 15(2), 145-164.

Marchal, S., Boukari, M. et Cayssials, J.L. (2007), L'impact des normes IFRS sur les données comptables des groupes français cotés, Bulletin de la Banque de France, Juillet, 163, 27-42

Pincus, M., Rajgopal, S. et Venkatachalam, M. (2007), The Accrual Anomaly: International Evidence, The Accounting review, 82(1), 169-203.

Warfield, T. et Wild, J. (1992), Accounting Recognition and the Relevance of Earnings as an Explanatory Variable for Returns, The Accounting Review, 67 (4), 821-842. 